EEG power spectrum in eating and weight disorders

\title{
Abnormalities in the EEG power spectrum in bulimia nervosa, binge-eating disorder, and obesity: A systematic review
}

Marie Blume, Ricarda Schmidt, Anja Hilbert

\begin{abstract}
Affiliation:
Integrated Research and Treatment Center AdiposityDiseases, Departments of Medical Psychology and Medical Sociology and Psychosomatic Medicine and Psychotherapy, University of Leipzig Medical Center, Germany
\end{abstract}

\section{Corresponding author:}

Marie Blume, M.Sc., Integrated Research and Treatment Center AdiposityDiseases, Departments of Medical Psychology and Medical Sociology and Psychosomatic Medicine and Psychotherapy, University of Leipzig Medical Center, Philipp-Rosenthal-Strasse 27, 04103 Leipzig, Germany. Phone: +49 341 97-15380, Fax: +49 341 97-15359, Email: marie.blume@medizin.uni-leipzig.de

Running title: EEG power spectrum in eating and weight disorders

\section{Conflict of interest statement}

The authors declare that they have no conflict of interest.

\section{Acknowledgements}

This study was supported by the Federal Ministry of Education and Research, Germany (Grant 01EO1501).

The final publication is available at https://doi.org/10.1002/erv.2654 
EEG power spectrum in eating and weight disorders

\begin{abstract}
To provide a basis for electroencephalography (EEG) neurofeedback protocols for bulimia nervosa $(\mathrm{BN})$, binge-eating disorder (BED), and obesity, this systematic review investigates alterations in EEG-measured brain activity, specifically frequency bands. A systematic literature search with pre-defined search terms yielded $N=7$ studies meeting the inclusion criteria. The risk of bias was assessed for all studies. In resting-state EEG, the beta activity was elevated in fronto-central regions in individuals with obesity and comorbid BED. In food-cue conditions, both obese individuals with and without BED showed increased beta activity, suggesting increased awareness of food cues and a heightened attentional focus towards food stimuli. The level of beta activity was positively correlated with eating disorder psychopathology in resting and food-cue conditions. In individuals with $\mathrm{BN}$, there was no evidence for altered EEG spectral power. The results indicate specific alterations in EEGbased brain activity in individuals with BED and obesity. More high-quality studies are needed to further confirm these findings and to transfer them into EEG-based interventions. Keywords: EEG power spectrum, binge-eating disorder, bulimia nervosa, obesity
\end{abstract}


EEG power spectrum in eating and weight disorders

\section{Introduction}

Conditions involving excess body weight, binge-eating, and overeating are highly prevalent, and although remission rates indicate that $50 \%$ of the treatment-seeking individuals with bulimia nervosa (BN), binge-eating disorder (BED), or obesity show an abatement of symptoms by evidence-based treatment options, a substantial proportion shows a maintenance or worsening of symptoms (Hay, 2013; Rudolph, Hellbardt, Baldofski, Zwaan, \& Hilbert, 2016; Vocks et al., 2010). BN is characterized by recurrent episodes of binge-eating, defined as consuming an unambiguously large amount of food in a discrete period of time while experiencing loss of control over eating, accompanied by extreme compensatory behaviors in order to prevent weight gain (American Psychiatric Association, 2013). Although recurrent binge-eating episodes are core features of BED as well, no regular inappropriate compensatory behaviors are shown in individuals with BED (American Psychiatric Association, 2013). In the general population, lifetime prevalences up to $0.8 \%$ and 3.6\% have been reported for BN and BED, respectively (Smink, van Hoeken, Oldehinkel, \& Hoek, 2014; Solmi, Hotopf, Hatch, Treasure, \& Micali, 2016). Importantly, about $33 \%$ of individuals with $\mathrm{BN}$ and $88 \%$ of individuals with BED display a comorbid obesity (Villarejo et al., 2012), which is defined as an abnormal or excessive accumulation of body fat (body mass index, BMI $\geq 30.0 \mathrm{~kg} / \mathrm{m}^{2}$; World Health Organisation, 2017).

Recent evidence suggests that treatment success in BN, BED, and obesity might be impaired by general and, more importantly, food-specific self-regulatory deficits (e. g., Brockmeyer et al., 2016; Manasse et al., 2016; Sysko et al., 2017). A number of studies suggested that the recruitment of the prefrontal control circuitry is altered in these conditions, which might explain the diminished self-regulatory abilities in several executive functions, such as impulsivity, set-shifting, or inhibition (Lavagnino, Arnone, Cao, Soares, \& Selvaraj, 2016; Steward, Menchón, Jiménez-Murcia, Soriano-Mas, \& Fernández-Aranda, 2018). These 
EEG power spectrum in eating and weight disorders

alterations are particularly pronounced in response to food cues and present, for example, in a decreased activation of the prefrontal cortex in individuals with $\mathrm{BN}$ and those with obesity, and increased activation of the orbitofrontal cortex in individuals with BED as compared to healthy individuals (Friederich, Wu, Simon, \& Herzog, 2013; Lavagnino et al., 2016). Coupled with an attentional food bias, increased reactivity to food cues and increased impulsivity, individuals with BN, BED, and obesity may have a neuropsychological profile, which is prone to increased food intake and overeating (Brockmeyer et al., 2016; Giel, Speer, Schag, Leehr, \& Zipfel, 2017; Pearson, Wonderlich, \& Smith, 2015; Stojek et al., 2018; I. Wolz, Fagundo, Treasure, \& Fernandez-Aranda, 2015). Therefore, neurophysiological interventions are thought to be valuable assets in the treatment of underlying neurophysiological alterations of self-regulatory deficits in BN, BED, and obesity (Dalton, Campbell, \& Schmidt, 2017; Val-Laillet et al., 2015).

In the context of mental disorders characterized by increased impulsivity and decreased inhibition (e.g., attention-deficit/hyperactivity disorder [ADHD] and substance use disorders), electroencephalography (EEG) neurofeedback has been used to treat predefined EEG abnormalities in children and adults, leading to improved neural activity and less impulsive and disinhibited behavior (Arns, Heinrich, \& Strehl, 2014; Baumeister et al., 2018; Bluschke, Broschwitz, Kohl, Roessner, \& Beste, 2016; Sokhadze, Cannon, \& Trudeau, 2008). EEG neurofeedback is based on the spectral analysis of the EEG, which enables the digital representation of specific frequency bands (e.g., delta, theta, alpha, beta) with high temporal resolution (Andreassi, 2006). Most commonly a fast Fourier transformation is used to determine the relative or absolute power of the frequency bands over certain cortical areas for a brief amount of time (Demos, 2005). Power changes in specific frequency bands may be an indicator for an increased firing rate within certain cell populations reflecting different stages of cognitive arousal, cognitive processing and psychopathology (Demos, 2005; 
EEG power spectrum in eating and weight disorders

Klimesch, 1999; Makeig, Debener, Onton, \& Delorme, 2004) as depicted in Table 1. For example, the beta band can be divided in low and high beta activity. While low beta $(12-15$ $\mathrm{Hz}$ ) is mostly associated with quiet, focused, and introverted concentration, high beta (21 $32 \mathrm{~Hz}$ ) is commonly related to significant stress, anxiety, paranoia, high energy, and high arousal (Abhang, Gawali, \& Mehrotra, 2016; Demos, 2005). Clinically, abnormalities in the beta band are characteristic for individuals with impulse regulation disorders (e.g., substance use disorders; Knott, Cosgrove, et al., 2008; Knott, Naccache, et al., 2008; Littel, Franken, \& Van Strien, 2009; Liu, Vaupel, Grant, \& London, 1998; Reid et al., 2003).

- Please insert Table 1 here -

Existing systematic and non-systematic reviews investigating the relationship between brain functions, cognitive functions, and eating behavior in $\mathrm{BN}, \mathrm{BED}$, and obesity focused on findings from neuroimaging studies using functional magnetic resonance imaging (fMRI), magnetic resonance imaging, positron emission tomography (Carnell, Gibson, Benson, Ochner, \& Geliebter, 2012; Kessler, Hutson, Herman, \& Potenza, 2016; Lavagnino et al., 2016; Steward et al., 2018), or analyzed event-related potentials (ERPs) and sleep EEG (Jáuregui-Lobera, 2011; I. Wolz et al., 2015). However, these reviews did not provide information on abnormalities in the EEG power spectrum in BN, BED, and obesity, which is, however, a prerequisite for the development of EEG neurofeedback paradigms, targeting disorder-specific abnormalities in the EEG power spectrum in these conditions. Therefore, this review sought to systematically examine abnormalities in the EEG power spectrum in resting-state EEG and/or during general and/or food-cue exposure in individuals with $\mathrm{BN}$, BED, and obesity.

\section{Method}

\section{Search and Study Selection}


EEG power spectrum in eating and weight disorders

This systematic review was conducted according to the PRSIMA-P guidelines (Moher et al., 2015). Relevant studies published before June 2018 were identified through six electronic databases: PubMed, PsychInfo, Web of Science, Pubpsych, Lilacs, and PsychArticles. The following search terms were used to identify relevant terms in title, abstract, and keywords (or full text): (brainwave OR electroencephalography OR EEG OR qeeg) AND ((eating disorder*) OR obes* OR overweight OR adipos* OR (binge eat*) OR bulimi* OR (loss of control)). Additionally, reference lists of included articles and reviews were scanned for relevant studies. Duplicates were removed and full-text articles from all relevant titles and abstracts were screened according to pre-specified eligibility criteria.

Inclusion in the review required: (1) a diagnosis of $\mathrm{BN}, \mathrm{BED}$ according to an expert interview or self-report, and/or obesity, i.e., BMI $\geq 30.0 \mathrm{~kg} / \mathrm{m}^{2}$, as determined by objective measures of weight and height, and (2) outcomes using a spectral power analysis. Articles were excluded, if they (1) were written in any other language than German or English, (2) had a sample size $n<5$, (3) investigated ERPs only, sleep EEG, or disordered sleeping, or used LORETA source analysis only, or (4) were not original research reports.

\section{Data Extraction}

The extracted information was entered in a pre-defined extraction table, including sample size, eating disorder diagnosis, type of sample, assessments, electrode positions, as well as results on EEG power spectrum.

\section{Assessment of Study Quality and Risk of Bias}

The quality of the included studies and the potential risk of bias were assessed using the quality assessment tool for quantitative studies from the Effective Public Health Practice Project (EPHPP; National Collaborating Centre for Methods and Tools, 2009). The EPHPP can be used to determine the quality and the risk of bias in observational, before-and-after and case-control studies, as well as in randomized controlled trails. It considers six 
EEG power spectrum in eating and weight disorders

components: (1) selection bias, (2) study design, (3) confounders, (4) blinding, (5) data collection methods, and (6) withdrawals and dropouts. Every component receives a rating of 1 "strong," 2 "moderate," or 3 "weak risk of bias". Using the average of all ratings, each included study was assigned a weak, moderate, or strong quality. The EPHPP displays excellent inter-rater reliability of the total grade of rating and is therefore considered a valid assessment tool (Armijo-Olivo, Stiles, Hagen, Biondo, \& Cummings, 2012).

\section{Results}

The results of the systematic literature search are depicted in the PRISMA flow chart in Figure 1.

- Please insert Figure 1 here -

Out of the seven included studies investigating eight samples, two studies analyzed spectral power in individuals with BN (Léonard, Pepinà, Bond, \& Treasure, 1998; Wolz et al., 2017), three studies investigated individuals with BED (Imperatori et al., 2015; Tammela et al., 2010; Wolz et al., 2017), and three studies investigated individuals with obesity only (Bauer \& Manning, 2016; Hume, Howells, Karpul, et al., 2015; Hume, Howells, Rauch, Kroff, \& Lambert, 2015). All characteristics of included studies are summarized in Table 2.

- Please insert Table 2 here -

\section{Bulimia Nervosa}

Léonard et al. (1998) and Wolz et al. (2017) examined EEG brain activity in women with BN (American Psychiatric Association, 1994, 2013). BN was determined via clinical expert rating (Léonard et al., 1998) and a semi-structured expert interview (Wolz et al., 2017). Léonard et al. (1998) derived the EEG at two standard positions according to the international 10 - 20 system (Cz and C3) at 1-minute resting-state eyes-open before, during, and after a test meal. The test meal consisted of a midmorning snack of about $1350 \mathrm{~kJ}$ (Léonard et al., 1998). Wolz et al. (2017) derived the EEG from 60 electrode positions 
EEG power spectrum in eating and weight disorders

(10 - 20 system) and used four active electrodes (Fz, FCz, F1, F2) to determine changes in frontal theta activity during a 1-minute odor presentation with a neutral (pencil smell) and a chocolate (piece of chocolate) aroma, followed by a visual presentation of 56 neutral (office items) or chocolate pictures.

Leonard et al. (1998) reported no differences in the delta, theta, alpha, or beta power between the three conditions and in comparison to a group of individuals with anorexia nervosa and a healthy control group. Wolz et al. (2017) did not detect differences in frontal theta activity between the olfactory or visual presentation of neutral and chocolate stimuli in women with BED. In addition, the BED group did not differ in frontal theta activity from a healthy normal weight control group in any of these conditions.

\section{Binge-Eating Disorder}

Two studies investigated adults with BED and comorbid obesity (American Psychiatric Association, 1994, 2000; Imperatori et al., 2015; Tammela et al., 2010) and one study investigated women with BED across the weight range (Wolz et al., 2017). In one study BED was determined via a semi-structured expert interview according to DSM-5 criteria (Wolz et al., 2017) and two studies (Imperatori et al., 2015; Tammela et al., 2010) used a self-report questionnaire (Binge Eating Scale [BES], Gormally, Black, Daston, \& Rardin, 1982). Imperatori et al. (2015) derived the resting-state EEG eyes-closed for 5 minutes at 32 standard positions (10 - 20 system), after a 4 - 6 hour fast. Tammela et al. (2010) derived the EEG from 64 electrode positions (10 - 20 system) after an overnight fast at resting-state eyesclosed for an unspecified amount of time and at resting-state eyes-open for 15 minutes each before a neutral control and a food-cue condition. In the control condition participants looked at a colorful landscape picture for approximately 33 minutes with breaks; in the food-cue condition they were presented with an individually selected, real, and freshly cooked meal for approximately 33 minutes with breaks (Tammela et al., 2010). As described above, Wolz et 
EEG power spectrum in eating and weight disorders

al. (2017) compared frontal theta activity between women with BED and a normal weight control group during an olfactory and visual observation task.

Individuals with BED displayed higher fronto-central beta activity in the resting-state eyes-closed EEG compared to individuals without BED (Imperatori et al., 2015; Tammela et al., 2010). In addition, the study by Tammela et al. (2010) found individuals with BED to display an elevation of beta power during both the food-cue and the control condition compared to individuals with obesity only. Furthermore, individuals of both groups displayed higher beta power during the food-cue condition compared to the control condition (Tammela et al., 2010). The beta activity of individuals with BED was positively correlated to BED symptomatology as indicated by the sum score of the BES and the severity and symptom score of the Bulimic Investigatory Test (Tammela et al., 2010). Compared to a healthy normal weight control group and women with $\mathrm{BN}$, frontal theta activity in response to olfactorily or visually presented neutral or chocolate stimuli did not differ in women with BED (Wolz et al., 2017).

\section{Obesity}

Two of three studies investigating obesity included otherwise healthy adults with obesity (BMI $\geq 30.0$ kg/m²; Hume, Howells, Karpul, et al., 2015; Hume, Howells, Rauch, et al., 2015) and one study investigated adolescent girls with a body mass index percentile $\geq 85$ (Bauer \& Manning, 2016; Hume, Howells, Karpul, et al., 2015; Hume, Howells, Rauch, et al., 2015). The EEG was derived from 10 (Hume, Howells, Karpul, et al., 2015; Hume, Howells, Rauch, et al., 2015) and 64 electrode positions (Bauer \& Manning, 2016) according to the international 10 - 20 system and was recorded during a modified Stroop task using office and food stimuli (Hume, Howells, Karpul, et al., 2015; Hume, Howells, Rauch, et al., 2015), and during a working memory and sustained attention task (Bauer \& Manning, 2016). The modified Stroop task used by Hume et al. (2015) was divided into three distinct subtests: 
EEG power spectrum in eating and weight disorders

A practice task, a control task, and a food-cue task (Hume, Howells, Karpul, et al., 2015; Hume, Howells, Rauch, et al., 2015). Each task included images which appeared at random between the standard Stroop word/color prompts while EEG was recorded. During the practice task, 20 grey squares, during the office task, 20 pictures of neutral, office-related items (e.g. ball-point pen, bookshelf, computer cables), and during the food-cue task, 20 pictures of pleasant, palatable foods (e.g., bonbons, bread buns, chocolate cake) randomly appeared in between the standard Stroop word/color prompts. Additionally to a reaction time task, participants were instructed to count and verbally recall the number of grey squares/office/food pictures afterwards. Bauer and Manning (2016) asked participants during a working memory task to memorize 3 - 7 visual objects in 5 seconds, which were previously presented in a $3 \times 4$ grid with 12 possible locations on the computer screen. During the attention task participants were asked to react to a rarely occurring pure $500 \mathrm{~Hz}$ tone as quickly as possible and to ignore frequent noise bursts (Bauer \& Manning, 2016). None of the studies investigated the resting-state EEG.

During the food-cue task, a significant elevation of frontal beta power was found in women with obesity compared to an age-matched normal weight control group (Hume, Howells, Rauch, et al., 2015). However, no differences in the EEG power spectrum were identified between women with obesity who had lost, but subsequently regained at least $10 \%$ of their body weight versus women with sustained obesity (Hume, Howells, Karpul, et al., 2015).

Comparisons between EEG spectral power in the food-cue versus office task were not provided. During the sustained attention task, adolescent girls with obesity displayed a significant elevation of frontal beta power compared to an age-matched normal weight control group (Bauer \& Manning, 2016). In the neutral office task women with obesity were characterized by significantly reduced prefrontal theta power compared to women with 
EEG power spectrum in eating and weight disorders

normal weight (Hume, Howells, Rauch, et al., 2015). Regarding clinical associations, heightened beta power over the frontal cortices during food-cue exposure (Hume, Howells, Rauch, et al., 2015) and during sustained attention (Bauer \& Manning, 2016) was positively related to percentage of body fat and the presence of symptoms of binge eating, BN, loss of control eating, and negatively correlated with body shape satisfaction.

\section{Assessment of Study Quality and Risk of Bias}

As depicted in Table 3, the overall study quality was medium, particularly due to the quasi-experimental nature of all studies investigating EEG spectral power, including the lack of random allocation to $\mathrm{BN}, \mathrm{BED}$, obese, or healthy control, and the unclear status of blinding of assessors and/or participants. One study was classified with weak study quality (Tammela et al., 2010), since depression was not included as a potential confounder in statistical analyses, despite observed group differences. One major limitation to the study quality in studies examining BED was the diagnostic method, consisting of self-administered questionnaires instead of a clinical interview. In addition, four studies (Hume, Howells, Karpul, et al., 2015; Hume, Howells, Rauch, et al., 2015; Imperatori et al., 2015; Wolz et al., 2017) addressed a possible alpha error accumulation.

- Please insert Table 3 here -

\section{Discussion}

In light of growing research in the area of neurophysiological processes in eating and weight disorders, this systematic review synthesized evidence on abnormalities in the EEG power spectrum in individuals with $\mathrm{BN}, \mathrm{BED}$, and obesity. The results indicated the presence of EEG abnormalities in individuals with BED during resting-state and food-cue conditions and in individuals with obesity during food-cue conditions only, while evidence in individuals with $\mathrm{BN}$ was scarce. 
EEG power spectrum in eating and weight disorders

EEG spectral activity in BN has only been investigated in two studies (Léonard et al., 1998; Wolz et al., 2017). In comparison with healthy individuals with normal weight and individuals with anorexia nervosa (Léonard et al., 1998), and individuals with BED (Wolz et al., 2017) those with BN did not differ regarding the spectral EEG activity before, during, or after a test meal (Léonard et al., 1998) or in response to olfactory or visual chocolate stimuli (Wolz et al., 2017). In contrast, previous fMRI studies in individuals with BN observed altered activity in the prefrontal cortex during food-cue exposure, which was positively associated with inhibitory control measured during neuropsychological tests (Friederich et al., 2013; Hirst et al., 2017). However, several methodological weaknesses of the study by Léonard et al. (e.g., small sample, concurrent psychotherapy, and use of only two active electrodes) limit the comparability to other neuropsychological findings; therefore, further systematic research in the EEG power spectrum in individuals with BN is recommended.

\section{Altered activity in the beta band}

Individuals with obesity only and individuals with BED displayed heightened activity in the beta band $(14-30 \mathrm{~Hz})$ in fronto-central regions during food-cue conditions as well as in sustained attention tasks compared to resting-state EEG (Bauer \& Manning, 2016; Hume, Howells, Rauch, et al., 2015; Tammela et al., 2010). Oscillations in the beta band are associated with conscious precision, strong focus, and ability to solve problems (Marzbani, Marateb, \& Mansourian, 2016), but also with a heightened state of alertness (Demos, 2005). Substances used to arouse concentration, focus, and alertness (e.g., Ritalin or Adderall) were found to be associated with heightened beta band activity as well (Marzbani et al., 2016). Although in healthy adults reward-related stimuli, such as monetary rewards, are related to elevated high beta activity $(20-35 \mathrm{~Hz})$ in fronto-central regions (Hosseini \& Holroyd, 2015), suggesting elevated high beta activity being a normative reaction to rewarding stimuli, individuals with BED showed a stronger elevation of high beta activity than those without 
EEG power spectrum in eating and weight disorders

BED. In addition, the present results are in line with findings from research in individuals with substance use disorders, demonstrating that the confrontation with the addictive substance (e.g., alcohol, cocaine, cigarettes) was accompanied by an elevation of beta band activity (Knott, Cosgrove, et al., 2008; Knott, Naccache, et al., 2008; Littel, Franken, \& Van Strien, 2009; Liu, Vaupel, Grant, \& London, 1998; Reid et al., 2003). Therefore, the findings might relate to an increased awareness of food cues and a heightened attentional focus towards food stimuli in individuals with obesity and individuals with BED (Field et al., 2016). These results complement findings from neuroimaging studies (mostly fMRI), proposing a relationship between lower prefrontal cortex activity, general and food-related inhibitory control, and higher BMI in individuals with obesity and/or BED (Lavagnino et al., 2016). Even after successful weight loss women with former obesity still demonstrated a heightened activity in the beta band in response to food cues (Hume, Howells, Karpul, et al., 2015), suggesting that body weight alone might not account for the altered brain activity, or that long-term excess body weight may alter the neurophysiological profile on a long-term basis.

While individuals with obesity presented with an elevation in beta activity only during food-cue and sustained attention tasks, individuals with obesity and comorbid BED furthermore presented with heightened beta power during resting-state EEG (Hume, Howells, Rauch, et al., 2015; Imperatori et al., 2015; Tammela et al., 2010). The elevated beta activity in resting-state EEG in those with comorbid BED was related to symptoms of binge-eating, BN, loss of control eating, and anxiety (Tammela et al., 2010). Consistently, difficulties in executive functioning in individuals with BED as compared to those with obesity only were not only observed in food-related tasks, but in general executive functioning tasks as well (e.g., Manasse et al., 2015). In addition to an increased awareness of food cues and attentional focus towards food, the elevated beta activity during resting-state EEG suggests a 
EEG power spectrum in eating and weight disorders

heightened general stress level in individuals with BED (Demos, 2005). Interestingly, there is evidence for elevated beta activity in resting-state EEG in individuals with substance use disorders compared to healthy control groups (Sokhadze et al., 2008), suggesting a possible neuronal overlap of BED and substance use disorders. Strikingly, elevated high beta activity during resting-state EEG was predictive of treatment failure in individuals with substance use disorders (e.g., cocaine, alcohol, heroin) compared to individuals maintaining abstinence, suggesting that elevated high beta activity may be an indicator of symptom severity (Sokhadze et al., 2008). Similarly, the heightened beta band activity in resting-state EEG might depict a maintenance mechanism of BED, leading to the above mentioned faster attentional engagement of food cues.

\section{Altered activity in the theta band}

Oscillations in the theta band are associated with working memory, executive functioning, and performance in inhibition tasks (Cavanagh \& Frank, 2014; Hsieh \& Ranganath, 2014; Kawasaki, Kitajo, \& Yamaguchi, 2010; Nigbur, Ivanova, \& Stürmer, 2011). Furthermore, it was found that in healthy individuals theta activity was decreased in response to different food odors (Krbot Skorić et al., 2015). Nevertheless, for individuals with $\mathrm{BN}$ and $\mathrm{BED}$ no alteration in frontal theta activity during the presentation with olfactory and visual chocolate stimuli versus neutral stimuli and versus a healthy normal weight control group were revealed (Wolz et al., 2017), suggesting that theta activity in BN and BED is not related to appetitive learning or appraisal. Results should be interpreted with caution; as participants were allowed to close their eyes during olfactory presentation, the increase in alpha activity might have disguised the underlying theta activity (Wolz et al., 2017).

Individuals with obesity only versus individuals with normal weight experienced a reduction in frontal theta power during a neutral office task (Hume, Howells, Rauch, et al., 2015), supporting evidence for difficulties in executive functioning in individuals with 
EEG power spectrum in eating and weight disorders

obesity (Gameiro, Perea, Ladera, Rosa, \& García, 2017). During a Go/ NoGo task, a neuropsychological inhibition task, frontal theta activity in healthy adults was strongest, when the need for inhibition was highest (e.g., incongruent trials; Nigbur et al., 2011). In frontal brain regions, theta activity is thought to play an active role in memory maintenance (Jensen \& Tesche, 2002). Current research found evidence that an EEG neurofeedback protocol aiming to enhance theta power led to improved memory consolidation in healthy adults (Reiner, Rozengurt, \& Barnea, 2014). One assumption to why frontal oscillations of the theta band are associated with enhanced cognitive functioning is that increased frontal theta power may reflect synchronous activity in brain regions that are involved in cognitively demanding tasks and is therefore enhanced when these brain regions are needed (Jensen \& Tesche, 2002; Onton, Delorme, \& Makeig, 2005). A reduction in frontal theta power in individuals with obesity during a cognitively demanding task could therefore underline deficits in executive functions (Gameiro et al., 2017).

\section{Strengths and Limitations}

With regard to the interpretation of presented findings, several strengths and limitations of the included studies have to be considered. First of all, the individual sample sizes of the included studies varied widely and were generally low, with most studies including less than 15 individuals in the experimental group. Second, in two studies investigating BED the BED diagnosis was derived from self-report only, potentially leading to misdiagnosis (Imperatori et al., 2015; Tammela et al., 2010). Third, two studies investigating BED included only individuals with comorbid obesity (Imperatori et al., 2015; Tammela et al., 2010), therefore, it remains unclear whether the identified EEG abnormalities apply to normal weight adults with BED as well. Fourth, although Wolz et al. (2017) included individuals with $\mathrm{BN}$ and BED across the weight range only theta activity was examined, leaving the question of possible differences in other frequency bands unanswered 
EEG power spectrum in eating and weight disorders

(Wolz et al., 2017). Fifth, two out of three studies on individuals with obesity did not assess possible comorbid eating disorders, leaving ambiguity about a possible interference of eating disorder symptomatology with EEG data (Hume, Howells, Karpul, et al., 2015; Hume, Howells, Rauch, et al., 2015). Finally, the study quality was mostly medium, due to the lack of information on blinding of participants/ assessors, the lack of random allocation to investigated groups, and lacking alpha error correction. Therefore, the presented results can only be seen as preliminary and require confirmation through further research.

Strengths of this systematic review include the systematic search strategy with an additive hand search, the systematic assessment of study outcomes, and evaluation of study quality. The inclusion of studies on clinical eating disorders and obesity only instead of subclinical eating disturbances enhanced the clinical relevance of our findings. However, the number of included studies was low $(N=7)$. Another limitation is the inclusion of articles published in German or English only, thus, relevant studies published in other languages have not been taken into account. Last, data extraction and the assessment of study quality were completed by the first author only.

\section{Conclusions and Future Directions}

The results of this systematic review indicated that individuals with obesity only and those with comorbid BED show altered activity in the EEG power spectrum. Alterations were most consistently reported for elevated beta activity, particularly during food-cue conditions. Only individuals with obesity and comorbid BED presented with an elevation in beta activity during resting-state EEG, suggesting a BED-specific EEG profile in the obesity spectrum. In contrast, no evidence for altered EEG spectral power was available for individuals with BN. Although EEG is not suited for precisely determining the origin or brain area of the derived signal, it is of note that included studies documented abnormalities over the frontal cortex, which coincided with the results of fMRI studies, suggesting altered brain activity in the 
EEG power spectrum in eating and weight disorders

prefrontal cortex of individuals with BED (Friederich et al., 2013; Hume, Howells, Karpul, et al., 2015; Hume, Howells, Rauch, et al., 2015; Imperatori et al., 2015; Kittel, Brauhardt, \& Hilbert, 2015; Lavagnino et al., 2016; Tammela et al., 2010).

This review is a first step for future investigations in the EEG power spectrum in $\mathrm{BN}$, BED, and obesity. To further distinguish between weight and eating disorder-related abnormalities, it would be beneficial to include individuals with obesity only, those with obesity and comorbid BED, and normal weight individuals with BED as well. Due to the lack of research on EEG spectral power in individuals with $\mathrm{BN}$, future research on neurophysiological alterations in BN is highly recommended. Longitudinal studies could provide further information about the stability of EEG alterations in individuals with BED and obesity. Such studies may reveal whether the abnormalities in the EEG power spectrum represent a prognostic indicator of symptom remission or treatment success in these populations.

Clinically, EEG neurofeedback seems to be a valuable addition to the treatment of individuals with BN, BED, and obesity given that recent studies in samples with subclinical eating disturbances revealed positive outcomes of EEG neurofeedback, specifically the reduction of food craving and subjective binge-eating tendencies (Schmidt \& Martin, 2015, 2016). Therefore, further research into treatment effects of EEG neurofeedback in BN, BED, and obesity is indicated (Bartholdy, Musiat, Campbell, \& Schmidt, 2013). 
EEG power spectrum in eating and weight disorders

\section{References}

Abhang, P. A., Gawali, B. W., \& Mehrotra, S. C. (2016). Chapter 3 - Technical aspects of brain rhythms and speech parameters. In P. A. Abhang, B. W. Gawali, \& S. C. Mehrotra (Eds.), Introduction to EEG-and speech-based emotion recognition (pp. 51-79). Academic Press. https://doi.org/10.1016/B978-0-12-804490-2.00003-8

American Psychiatric Association. (1994). DSM-IV: Diagnostic and statistical manual of mental disorders (4th edition). Arlington, VA: American Psychiatric Publishing.

American Psychiatric Association (2013). DSM-5: Diagnostic and statistical manual of mental disorders (5th edition). Arlington, VA: American Psychiatric Publishing.

Andreassi, J. L. (2006). Psychophysiology: human behavior and physiological response (5 revised edition). New York, NY: Taylor \& Francis Inc.

Armijo-Olivo, S., Stiles, C. R., Hagen, N. A., Biondo, P. D., \& Cummings, G. G. (2012). Assessment of study quality for systematic reviews: a comparison of the cochrane collaboration risk of bias tool and the effective public health practice project quality assessment tool: methodological research. Journal of Evaluation in Clinical Practice, 18, 12-18. https://doi.org/10.1111/j.1365-2753.2010.01516.x

Arns, M., Heinrich, H., \& Strehl, U. (2014). Evaluation of neurofeedback in ADHD: the long and winding road. Biological Psychology, 95, 108-115. https://doi.org/10.1016/j.biopsycho.2013.11.013

Bartholdy, S., Musiat, P., Campbell, I. C., \& Schmidt, U. (2013). The potential of neurofeedback in the treatment of eating disorders: a review of the literature. European Eating Disorders Review, 21, 456-463.

Bauer, L. O., \& Manning, K. J. (2016). Challenges in the detection of working memory and attention decrements among overweight adolescent girls. Neuropsychobiology, 73, 43-51. https://doi.org/10.1159/000442670 
EEG power spectrum in eating and weight disorders

Baumeister, S., Wolf, I., Holz, N., Boecker-Schlier, R., Adamo, N., Holtmann, M., ... Brandeis, D. (2018). Neurofeedback training effects on inhibitory brain activation in adhd: a matter of learning? Neuroscience, 378, 89-99.

https://doi.org/10.1016/j.neuroscience.2016.09.025

Bluschke, A., Broschwitz, F., Kohl, S., Roessner, V., \& Beste, C. (2016). The neuronal mechanisms underlying improvement of impulsivity in ADHD by theta/beta neurofeedback. Scientific Reports, 6, 31178. https://doi.org/10.1038/srep31178

Brockmeyer, T., Hamze Sinno, M., Skunde, M., Wu, M., Woehning, A., Rudofsky, G., \& Friederich, H.-C. (2016). Inhibitory control and hedonic response towards food interactively predict success in a weight loss programme for adults with obesity. Obesity Facts, 9, 299-309. https://doi.org/10.1159/000447492

Carnell, S., Gibson, C., Benson, L., Ochner, C. N., \& Geliebter, A. (2012). Neuroimaging and obesity: current knowledge and future directions. Obesity Reviews, 13, 43-56. https://doi.org/10.1111/j.1467-789X.2011.00927.x

Cavanagh, J. F., \& Frank, M. J. (2014). Frontal theta as a mechanism for cognitive control. Trends in Cognitive Sciences, 18, 414-421. https://doi.org/10.1016/j.tics.2014.04.012

Dalton, B., Campbell, I. C., \& Schmidt, U. (2017). Neuromodulation and neurofeedback treatments in eating disorders and obesity. Current Opinion in Psychiatry, 30, 458473. https://doi.org/10.1097/YCO.0000000000000361

Demos, J. N. (2005). Getting Started with Neurofeedback. New York: Ww Norton \& Co.

Field, M., Werthmann, J., Franken, I., Hofmann, W., Hogarth, L., \& Roefs, A. (2016). The role of attentional bias in obesity and addiction. Health Psychology, 35, 767-780. https://doi.org/10.1037/hea0000405 
EEG power spectrum in eating and weight disorders

Friederich, H.-C., Wu, M., Simon, J. J., \& Herzog, W. (2013). Neurocircuit function in eating disorders. International Journal of Eating Disorders, 46, 425-432. https://doi.org/10.1002/eat.22099

Gameiro, F., Perea, M. V., Ladera, V., Rosa, B., \& García, R. (2017). Executive functioning in obese individuals waiting for clinical treatment. Psicothema, 29, 61-66. https://doi.org/10.7334/psicothema2016.202

Giel, K. E., Teufel, M., Junne, F., Zipfel, S., \& Schag, K. (2017). Food-related impulsivity in obesity and binge eating disorder-a systematic update of the evidence. Nutrients, 9 . pii: E1170. https://doi.org/10.3390/nu9111170

Gormally, J., Black, S., Daston, S., \& Rardin, D. (1982). The assessment of binge eating severity among obese persons. Addictive Behaviors, 7, 47-55.

Hay, P. (2013). A systematic review of evidence for psychological treatments in eating disorders: 2005-2012. International Journal of Eating Disorders, 46, 462-469. https://doi.org/10.1002/eat.22103

Hirst, R. B., Beard, C. L., Colby, K. A., Quittner, Z., Mills, B. M., \& Lavender, J. M. (2017). Anorexia nervosa and bulimia nervosa: a meta-analysis of executive functioning. Neuroscience and Biobehavioral Reviews, 83, 678-690. https://doi.org/10.1016/j.neubiorev.2017.08.011

Hosseini, A. H., \& Holroyd, C. B. (2015). Reward feedback stimuli elicit high-beta EEG oscillations in human dorsolateral prefrontal cortex. Scientific Reports, 5. 13021. https://doi.org/10.1038/srep13021

Hsieh, L.-T., \& Ranganath, C. (2014). Frontal midline theta oscillations during working memory maintenance and episodic encoding and retrieval. NeuroImage, 85, 721-729. https://doi.org/10.1016/j.neuroimage.2013.08.003 
EEG power spectrum in eating and weight disorders

Hume, D. J., Howells, F. M., Karpul, D., Rauch, H. G. L., Kroff, J., \& Lambert, E. V. (2015). Cognitive control over visual food cue saliency is greater in reducedoverweight/obese but not in weight relapsed women: an EEG study. Eating Behaviors, 19, 76-80. https://doi.org/10.1016/j.eatbeh.2015.06.013

Hume, D. J., Howells, F. M., Rauch, H. G. L., Kroff, J., \& Lambert, E. V. (2015). Electrophysiological indices of visual food cue-reactivity. Differences in obese, overweight and normal weight women. Appetite, 85, 126-137. https://doi.org/10.1016/j.appet.2014.11.012

Imperatori, C., Fabbricatore, M., Farina, B., Innamorati, M., Quintiliani, M. I., Lamis, D. A., ... Speranza, A. M. (2015). Alterations of EEG functional connectivity in resting state obese and overweight patients with binge eating disorder: a preliminary report. Neuroscience Letters, 607, 120-124. https://doi.org/10.1016/j.neulet.2015.09.026 Jáuregui-Lobera, I. (2012). Electroencephalography in eating disorders. Neuropsychiatric Disease and Treatment, 8, 1-11. https://doi.org/10.2147/NDT.S27302

Jensen, O., \& Tesche, C. D. (2002). Frontal theta activity in humans increases with memory load in a working memory task. European Journal of Neuroscience, 15, 1395-1399. https://doi.org/10.1046/j.1460-9568.2002.01975.x

Kawasaki, M., Kitajo, K., \& Yamaguchi, Y. (2010). Dynamic links between theta executive functions and alpha storage buffers in auditory and visual working memory. European Journal of Neuroscience, 31, 1683-1689. https://doi.org/10.1111/j.14609568.2010.07217.x

Kessler, R. M., Hutson, P. H., Herman, B. K., \& Potenza, M. N. (2016). The neurobiological basis of binge-eating disorder. Neuroscience and Biobehavioral Reviews, 63, 223238. https://doi.org/10.1016/j.neubiorev.2016.01.013 
EEG power spectrum in eating and weight disorders

Kittel, R., Brauhardt, A., \& Hilbert, A. (2015). Cognitive and emotional functioning in bingeeating disorder: a systematic review. International Journal of Eating Disorders, 48, 535-554. https://doi.org/10.1002/eat.22419

Klimesch, W. (1999). EEG alpha and theta oscillations reflect cognitive and memory performance: a review and analysis. Brain Research Reviews, 29, 169-195. https://doi.org/10.1016/S0165-0173(98)00056-3

Knott, V., Cosgrove, M., Villeneuve, C., Fisher, D., Millar, A., \& McIntosh, J. (2008). EEG correlates of imagery-induced cigarette craving in male and female smokers. Addictive Behaviors, 33, 616-621. https://doi.org/10.1016/j.addbeh.2007.11.006

Knott, V., Naccache, L., Cyr, E., Fisher, D. J., McIntosh, J. F., Millar, A. M., \& Villeneuve, C. M. (2008). Craving-induced EEG reactivity in smokers: effects of mood induction, nicotine dependence and gender. Neuropsychobiology, 58, 187-199. https://doi.org/10.1159/000201716

Krbot Skorić, M., Adamec, I., Jerbić, A. B., Gabelić, T., Hajnšek, S., \& Habek, M. (2015). Electroencephalographic response to different odors in healthy individuals: a promising tool for objective assessment of olfactory disorders. Clinical EEG and Neuroscience, 46, 370-376. https://doi.org/10.1177/1550059414545649

Lavagnino, L., Arnone, D., Cao, B., Soares, J. C., \& Selvaraj, S. (2016). Inhibitory control in obesity and binge eating disorder: a systematic review and meta-analysis of neurocognitive and neuroimaging studies. Neuroscience \& Biobehavioral Reviews, 68, 714-726. https://doi.org/10.1016/j.neubiorev.2016.06.041

Lavender, J. M., \& Mitchell, J. E. (2015). Eating disorders and their relationship to impulsivity. Current Treatment Options in Psychiatry, 2, 394-401. https://doi.org/10.1007/s40501-015-0061-6 
EEG power spectrum in eating and weight disorders

Léonard, T., Pepinà, C., Bond, A., \& Treasure, J. (1998). Assessment of test-meal induced autonomic arousal in anorexic, bulimic and control females. European Eating Disorders Review, 6, 188-200. https://doi.org/10.1002/(SICI)10990968(199809)6:3<188::AID-ERV227>3.0.CO;2-G

Littel, M., Franken, I. H. A., \& Van Strien, J. W. (2009). Changes in the electroencephalographic spectrum in response to smoking cues in smokers and exsmokers. Neuropsychobiology, 59, 43-50. https://doi.org/10.1159/000205517

Liu, P. D., Xiang, Vaupel, P. D., D. Bruce, Grant, P. D., Steven, \& London, P. D., Edythe D. (1998). Effect of cocaine-related environmental stimuli on the spontaneous electroencephalogram in polydrug abusers. Neuropsychopharmacology, 19, 10-17. https://doi.org/10.1016/S0893-133X(97)00192-9

Makeig, S., Debener, S., Onton, J., \& Delorme, A. (2004). Mining event-related brain dynamics. Trends in Cognitive Sciences, 8, 204-210. https://doi.org/10.1016/j.tics.2004.03.008

Manasse, S. M., Espel, H. M., Schumacher, L. M., Kerrigan, S. G., Zhang, F., Forman, E. M., \& Juarascio, A. S. (2016). Does impulsivity predict outcome in treatment for binge eating disorder? A multimodal investigation. Appetite, 105, 172-179. https://doi.org/10.1016/j.appet.2016.05.026

Manasse, S. M., Forman, E. M., Ruocco, A. C., Butryn, M. L., Juarascio, A. S., \& Fitzpatrick, K. K. (2015). Do executive functioning deficits underpin binge eating disorder? A comparison of overweight women with and without binge eating pathology. International Journal of Eating Disorders, 48, 677-683. https://doi.org/10.1002/eat.22383 
EEG power spectrum in eating and weight disorders

Marzbani, H., Marateb, H. R., \& Mansourian, M. (2016). Neurofeedback: a comprehensive review on system design, methodology and clinical applications. Basic and Clinical Neuroscience, 7, 143-158. https://doi.org/10.15412/J.BCN.03070208

Moher, D., Shamseer, L., Clarke, M., Ghersi, D., Liberati, A., Petticrew, M., ... Stewart, L. A. (2015). Preferred reporting items for systematic review and meta-analysis protocols (PRISMA-P) 2015 statement. Systematic Reviews, 4, 1. https://doi.org/10.1186/2046-4053-4-1

Nigbur, R., Ivanova, G., \& Stürmer, B. (2011). Theta power as a marker for cognitive interference. Clinical Neurophysiology, 122, 2185-2194. https://doi.org/10.1016/j.clinph.2011.03.030

Onton, J., Delorme, A., \& Makeig, S. (2005). Frontal midline EEG dynamics during working memory. NeuroImage, 27, 341-356. https://doi.org/10.1016/j.neuroimage.2005.04.014

Pearson, C. M., Wonderlich, S. A., \& Smith, G. T. (2015). A risk and maintenance model for bulimia nervosa: from impulsive action to compulsive behavior. Psychological Review, 122, 516-535. https://doi.org/10.1037/a0039268

Reid, M. S., Prichep, L. S., Ciplet, D., O’Leary, S., Tom, M., Howard, B., ... John, E. R. (2003). Quantitative electroencephalographic studies of cue-induced cocaine craving quantitative electroencephalographic studies of cue-induced cocaine craving. Clinical Electroencephalography, 34, 110-123. https://doi.org/10.1177/155005940303400305

Reiner, M., Rozengurt, R., \& Barnea, A. (2014). Better than sleep: theta neurofeedback training accelerates memory consolidation. Biological Psychology, 95, 45-53. https://doi.org/10.1016/j.biopsycho.2013.10.010

Rudolph, A., Hellbardt, M., Baldofski, S., de Zwaan, M., \& Hilbert, A. (2016). Evaluation des einjährigen multimodalen Therapieprogramms DOC WEIGHT® 1.0 zur 
EEG power spectrum in eating and weight disorders

Gewichtsreduktion bei Patienten mit Adipositas Grad II und III. Psychotherapie, Psychosomatik, Medizinische Psychologie, 66, 316-323. https://doi.org/10.1055/s0042-110092

Schmidt, J., \& Martin, A. (2015). Neurofeedback reduces overeating episodes in female restrained eaters: a randomized controlled pilot-study. Applied Psychophysiology and Biofeedback, 40, 283-295. https://doi.org/10.1007/s10484-015-9297-6

Schmidt, J., \& Martin, A. (2016). Neurofeedback against binge eating: a randomized controlled trial in a female subclinical threshold sample. European Eating Disorders Review, 24, 406-416. https://doi.org/10.1002/erv.2453

Smink, F. R. E., van Hoeken, D., Oldehinkel, A. J., \& Hoek, H. W. (2014). Prevalence and severity of DSM-5 eating disorders in a community cohort of adolescents. International Journal of Eating Disorders, 47, 610-619. https://doi.org/10.1002/eat.22316

Sokhadze, T. M., Cannon, R. L., \& Trudeau, D. L. (2008). EEG biofeedback as a treatment for substance use disorders: review, rating of efficacy, and recommendations for further research. Applied Psychophysiology and Biofeedback, 33, 1-28. https://doi.org/10.1007/s10484-007-9047-5

Solmi, F., Hotopf, M., Hatch, S. L., Treasure, J., \& Micali, N. (2016). Eating disorders in a multi-ethnic inner-city UK sample: prevalence, comorbidity and service use. Social Psychiatry and Psychiatric Epidemiology, 51, 369-381. https://doi.org/10.1007/s00127-015-1146-7

Steward, T., Menchón, J. M., Jiménez-Murcia, S., Soriano-Mas, C., \& Fernández-Aranda, F. (2018). Neural network alterations across eating disorders: a narrative review of fMRI studies. Current Neuropharmacology, 16, 1-13. https://doi.org/10.2174/1570159X15666171017111532 
EEG power spectrum in eating and weight disorders

Stojek, M., Shank, L. M., Vannucci, A., Bongiorno, D. M., Nelson, E. E., Waters, A. J., ... Tanofsky-Kraff, M. (2018). A systematic review of attentional biases in disorders involving binge eating. Appetite. Advance online publication. https://doi.org/10.1016/j.appet.2018.01.019

Sysko, R., Ojserkis, R., Schebendach, J., Evans, S. M., Hildebrandt, T., \& Walsh, B. T. (2017). Impulsivity and test meal intake among women with bulimia nervosa. Appetite, 112, 1-8. https://doi.org/10.1016/j.appet.2017.01.005

Tammela, L. I., Paakkonen, A., Karhunen, L. J., Karhu, J., Uusitupa, M. I. J., \& Kuikka, J. T. (2010). Brain electrical activity during food presentation in obese binge-eating women. Clinical Physiology and Functional Imaging, 30, 135-140. https://doi.org/10.1111/j.1475-097X.2009.00916.X

Val-Laillet, D., Aarts, E., Weber, B., Ferrari, M., Quaresima, V., Stoeckel, L. E., ... Stice, E. (2015). Neuroimaging and neuromodulation approaches to study eating behavior and prevent and treat eating disorders and obesity. NeuroImage, 8, 1-31. https://doi.org/10.1016/j.nicl.2015.03.016

Villarejo, C., Fernández-Aranda, F., Jiménez-Murcia, S., Peñas-Lledó, E., Granero, R., Penelo, E., ... Menchón, J. M. (2012). Lifetime obesity in patients with eating disorders: increasing prevalence, clinical and personality correlates. European Eating Disorders Review, 20, 250-254. https://doi.org/10.1002/erv.2166

Vocks, S., Tuschen-Caffier, B., Pietrowsky, R., Rustenbach, S. J., Kersting, A., \& Herpertz, S. (2010). Meta-analysis of the effectiveness of psychological and pharmacological treatments for binge eating disorder. International Journal of Eating Disorders, 43, 205-217. https://doi.org/10.1002/eat.20696 
EEG power spectrum in eating and weight disorders

Wolz, I., Fagundo, A. B., Treasure, J., \& Fernandez-Aranda, F. (2015). The processing of food stimuli in abnormal eating: a systematic review of electrophysiology. European Eating Disorders Review, 23, 251-261. https://doi.org/10.1002/erv.2366

Wolz, I., Sauvaget, A., Granero, R., Mestre-Bach, G., Baño, M., Martín-Romera, V., ... Fernández-Aranda, F. (2017). Subjective craving and event-related brain response to olfactory and visual chocolate cues in binge-eating and healthy individuals. Scientific Reports, 7, 41736. https://doi.org/10.1038/srep41736

World Health Organization (2017). Obesity and overweight: Fact sheet. Retrieved July 30, 2017 from http://www.who.int/mediacentre/factsheets/fs311/en/ 


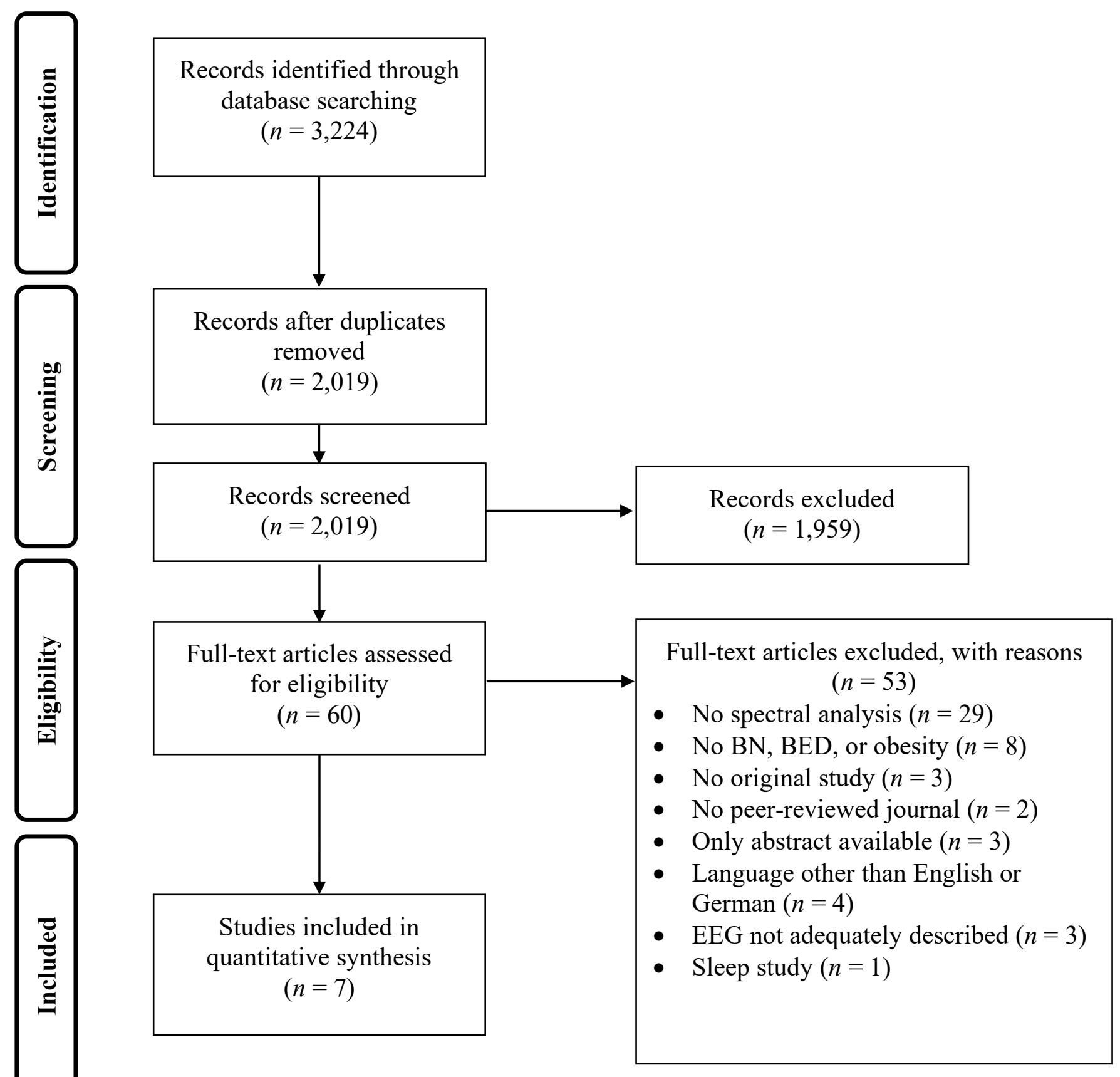

Figure 1. PRISMA flow chart

Note. BED: binge-eating disorder, BN: bulimia nervosa, EEG: electroencephalogram 
EEG power spectrum in eating and weight disorders

Table 1

Overview of frequency bands and associated cognitive states

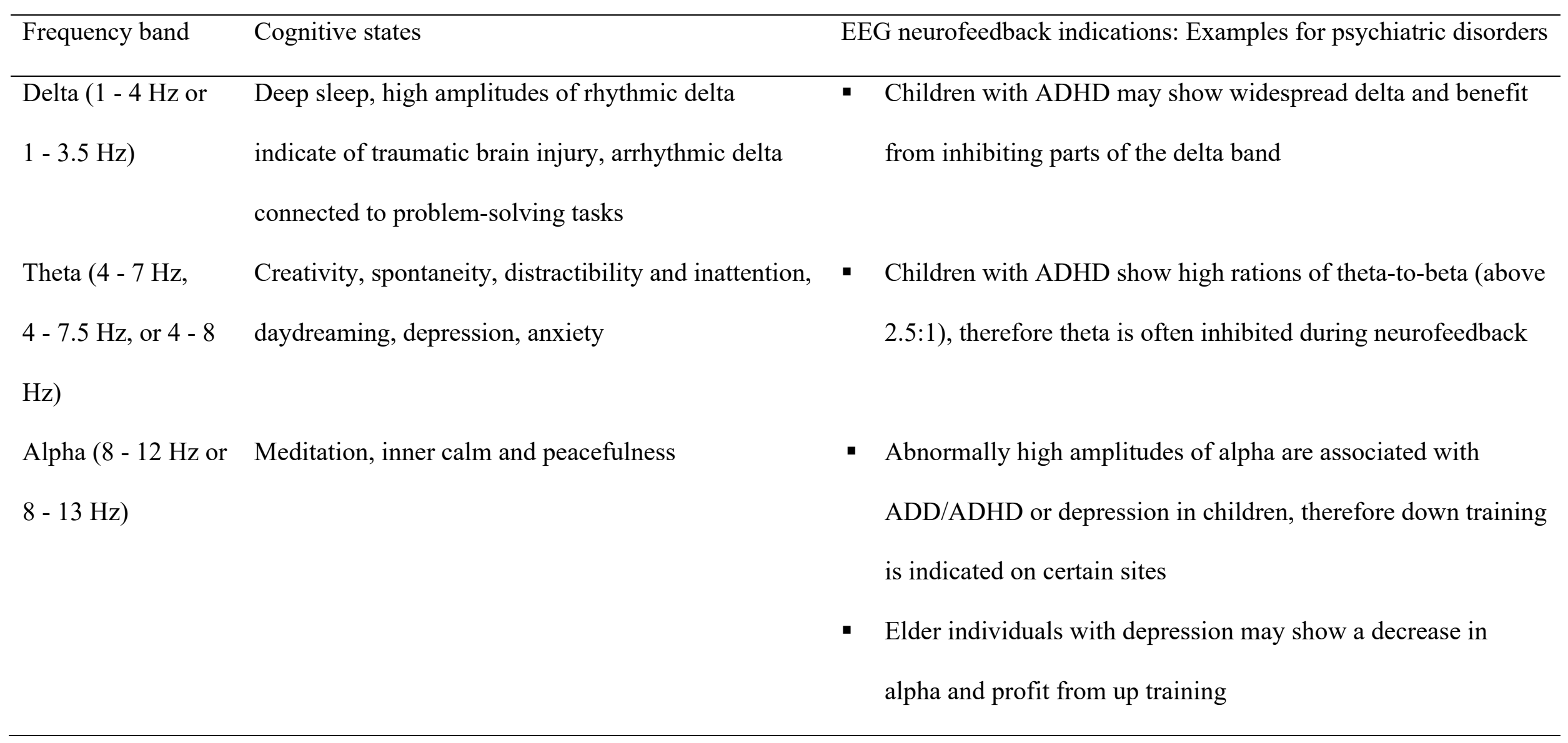


Table 1 continued

\begin{tabular}{lll}
\hline Frequency band $\quad$ Cognitive states & EEG neurofeedback indications: Examples for psychiatric \\
& disorders
\end{tabular}

Beta $(13-21 \mathrm{~Hz}) \quad$ Focused, analytical, relaxed thinking

Beta $(13-21 \mathrm{~Hz}) \quad$ Focused, analytical, relaxed thinking

High beta (21 - $32 \quad$ Peak performance, cognitive processing, worry,

Hz) anxiety, overthinking, rumination

Gamma (40 Hz or Problem-solving, learning, mental sharpness

$38-42 \mathrm{~Hz})$
- Excessive beta is found in many psychiatric disorders, e.g., in $\mathrm{ADD}$, OCD, sleep disorders, bruxism, learning disorders, anxiety disorders, depression

- Excessive high beta is associated with OCD, anxiety, alcoholism and insomnia, and therefore often inhibited

- Lack of $40 \mathrm{~Hz}$ bursts in individuals with learning disorders or mental deficits, advising a synchrony training

Note. ADHD: attention deficit and hyperactivity disorder, ADD: attention deficit disorder, OCD: obsessive compulsive disorder. Adapted from

“Getting Started with Neurofeedback” by J.N. Demos, 2005, New York: Ww Norton \& Co, p. 112 - 121 . Copyright 2005 by John N. Demos. 
EEG power spectrum in eating and weight disorders

\section{Table 2}

Studies investigating the EEG power spectrum

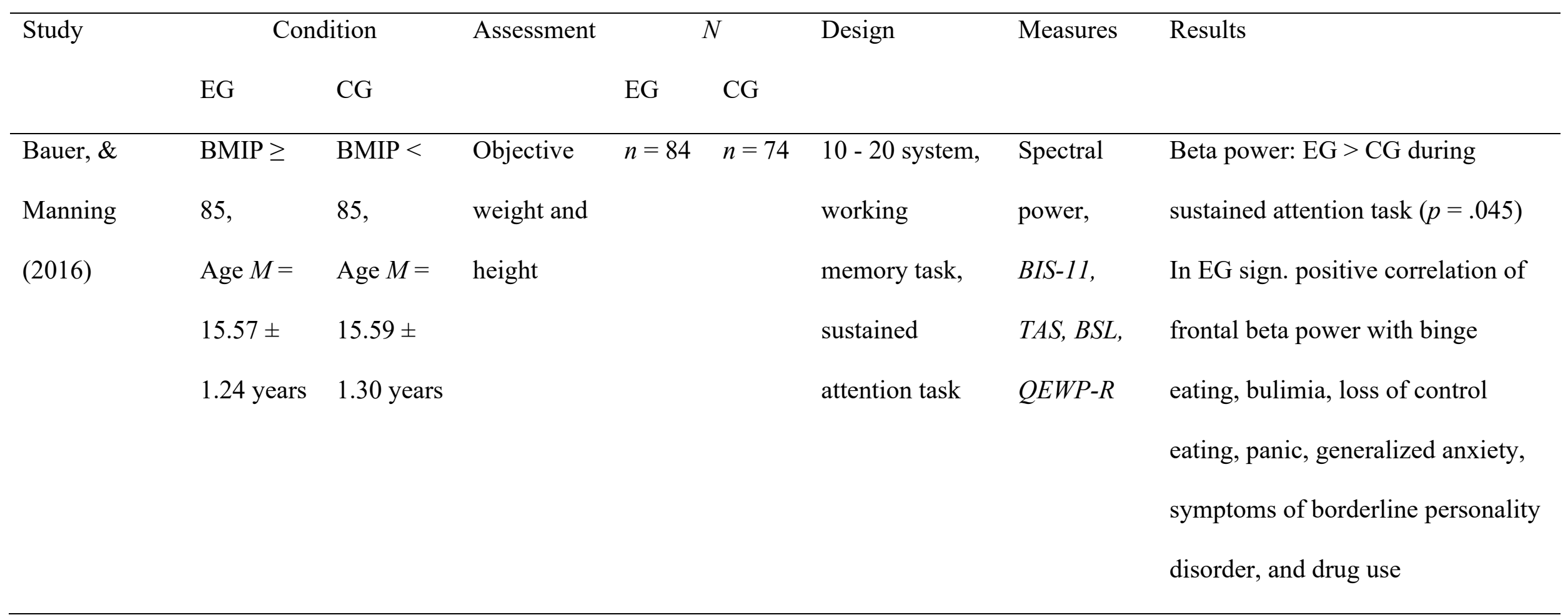


EEG power spectrum in eating and weight disorders

Table 2 (continued)

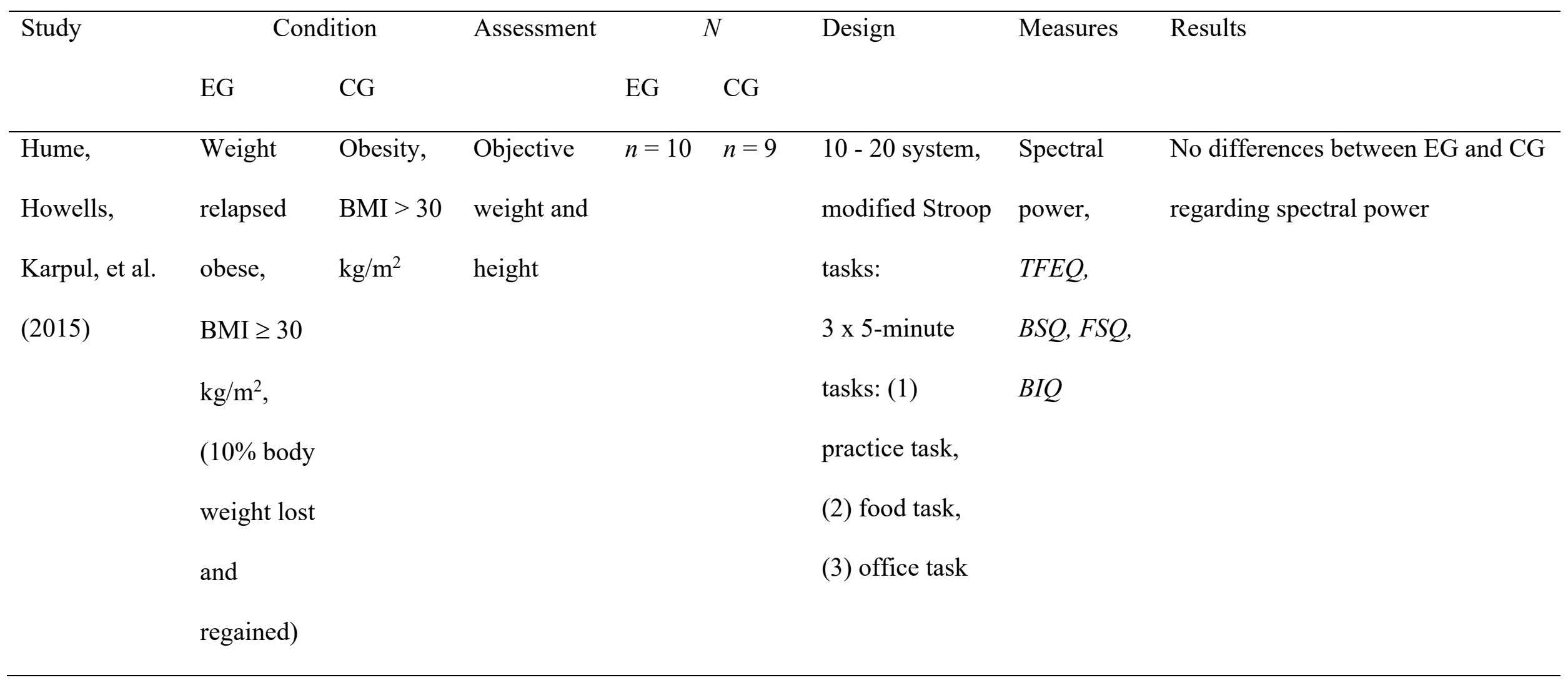


EEG power spectrum in eating and weight disorders

Table 2 (continued)

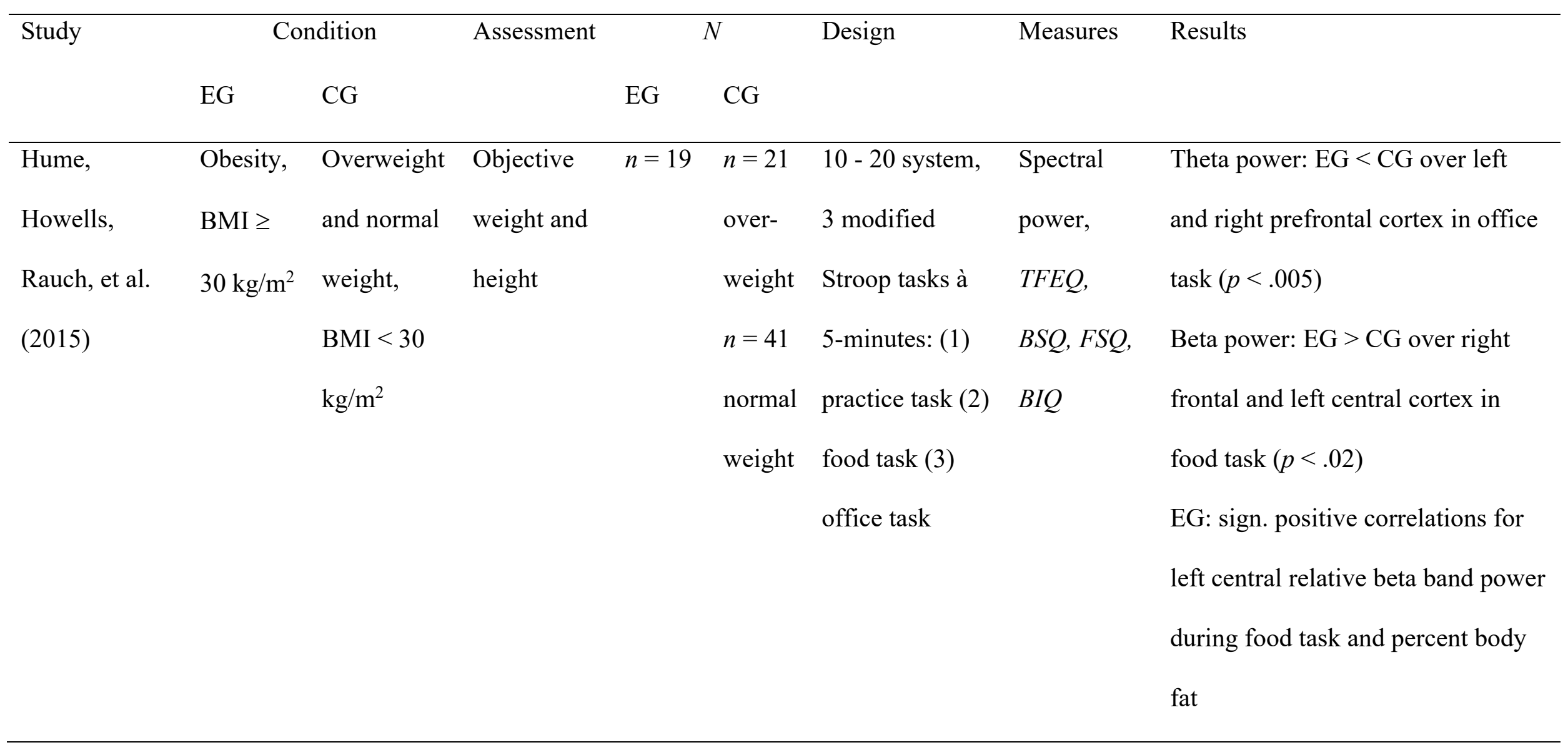


EEG power spectrum in eating and weight disorders

Table 2 (continued)

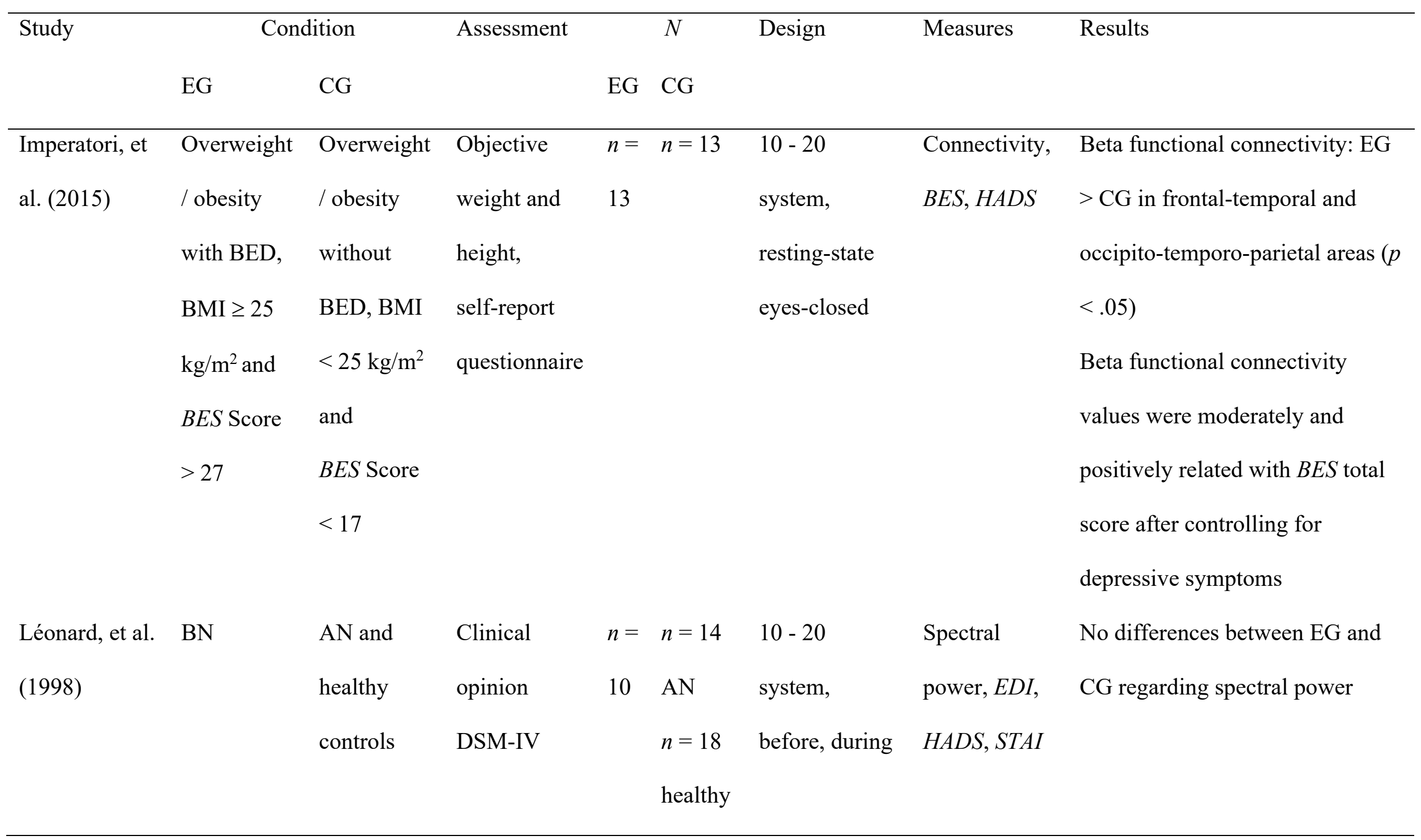


EEG power spectrum in eating and weight disorders

and after

test-meal 
EEG power spectrum in eating and weight disorders

Table 2 (continued)

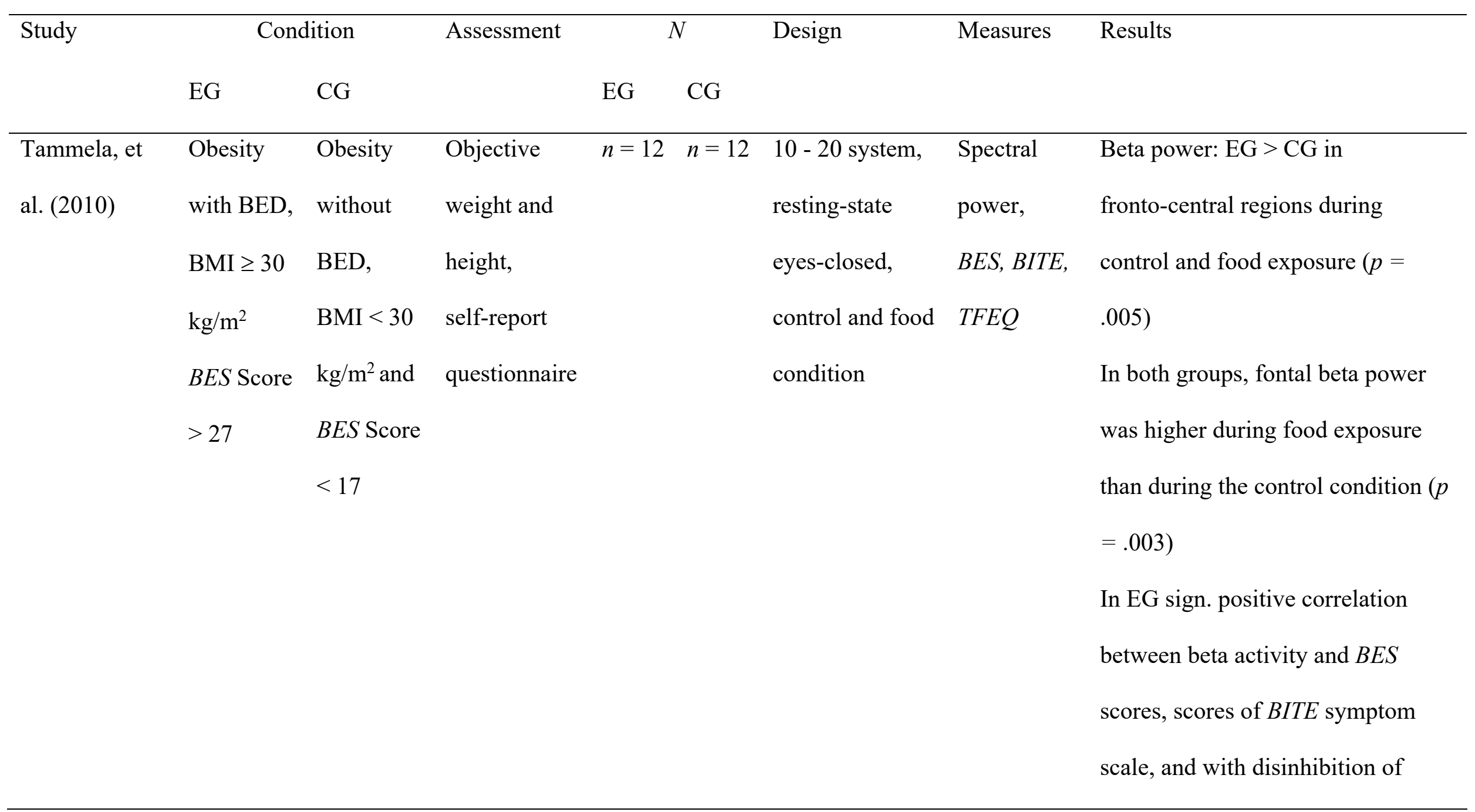


Table 2 (continued)

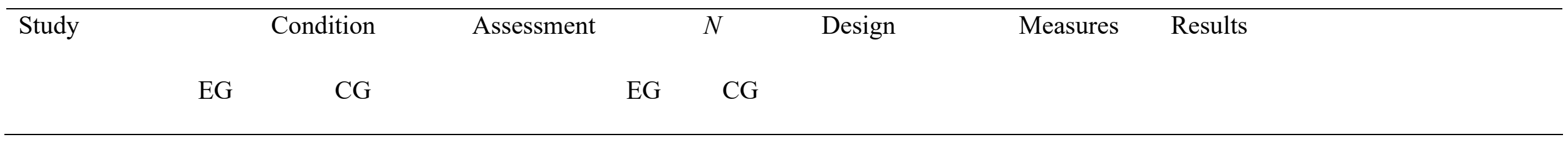




\begin{tabular}{|c|c|c|c|c|c|c|c|c|}
\hline Wolz et al. & BED and & Healthy & Objective & $n=7$ & $n=20$ & 10 - 20 system, & Theta & No differences between EGs and \\
\hline \multirow[t]{8}{*}{ (2017) } & $\mathrm{BN}$ & normal & weight and & BED & & olfactory and & power, & CG regarding frontal theta power \\
\hline & BMI $M=$ & weight & height, & $n=12$ & & visual control & ERPs, & during control and food conditions \\
\hline & 31.19 & controls & semi- & $\mathrm{BN}$ & & and food & FCCQ, & \\
\hline & $\mathrm{kg} / \mathrm{m}^{2} \pm$ & BMI $M=$ & structured & & & condition & DERS, & \\
\hline & 10.51 & 21.99 & expert & & & & YFAS-S, & \\
\hline & $\mathrm{kg} / \mathrm{m}^{2}$ & $\mathrm{~kg} / \mathrm{m}^{2} \pm$ & interview & & & & EDI-2, & \\
\hline & & 2.81 & & & & & SCL-90-R & \\
\hline & & $\mathrm{kg} / \mathrm{m}^{2}$ & & & & & & \\
\hline
\end{tabular}

Note. BN: bulimia nervosa, BED: binge-eating disorder, AN: anorexia nervosa, EG: experimental group, CG: control group, BMI: body mass index,

BMIP: body mass index percentile, BIS-11: Barratt Impulsiveness Scale, TAS: Toronto Alexithymia Scale, BSL: Borderline Symptom List,

QEWP-R: Revised Questionnaire of Eating and Weight Patterns, TFEQ: Three-Factor Eating Questionnaire, SFQ: Short Fat Questionnaire, BSQ:

Body Shape Questionnaire, BIQ: Body Image Questionnaire, BES: Binge Eating Scale, HADS: Hospital Anxiety and Depression Scale, EDI:

Eating Disorder Inventory, STAI: State-Trait Anxiety Inventory, BITE: Bulimic Investigatory Test, Edinburgh, ERPS: Event-Related Potentials,

FCCQ: Food Chocolate-Craving Questionnaire, DERS: Difficulties in Emotion Regulation Scale, YFAS-S: Yale Food Addiction Scale-Spanish

Version, EDI-2: Eating Disorder Inventory-2, SCL-90-R: Symptom Checklist-90-Revised. 
EEG power spectrum in eating and weight disorders 
EEG power spectrum in eating and weight disorders

Table 3

Quality and risk of bias assessment

\begin{tabular}{|c|c|c|c|c|c|c|c|c|}
\hline Study & $\begin{array}{l}\text { Selection } \\
\text { bias }\end{array}$ & $\begin{array}{l}\text { Study } \\
\text { design }\end{array}$ & Confounders & Blinding & $\begin{array}{l}\text { Data } \\
\text { collection } \\
\text { method }\end{array}$ & $\begin{array}{l}\text { Withdrawals } \\
\text { and dropouts }\end{array}$ & Overall & Note \\
\hline $\begin{array}{l}\text { Bauer \& Manning } \\
\text { (2016) }\end{array}$ & 2 & 3 & 1 & 2 & 3 & 1 & 2 & No alpha error correction \\
\hline $\begin{array}{l}\text { Hume, Howells, Karpul } \\
\text { et al. (2015) }\end{array}$ & 2 & 3 & 1 & 2 & 2 & 1 & 2 & \\
\hline $\begin{array}{l}\text { Hume, Howells, Rauch, } \\
\text { et al. (2015) }\end{array}$ & 2 & 3 & 1 & 2 & 2 & 1 & 2 & \\
\hline Tammela, et al. (2010) & 3 & 3 & 3 & 2 & 3 & 1 & 3 & $\begin{array}{l}\text { BED assessed using self-report, } \\
\text { No alpha error correction }\end{array}$ \\
\hline Wolz, et al. (2017) & 1 & 3 & 1 & 2 & 1 & 1 & 2 & \\
\hline
\end{tabular}


EEG power spectrum in eating and weight disorders

Note. Assessed using the quality assessment tool for quantitative studies from the Effective Public Health Practice Project (National Collaborating

Centre for Methods and Tools, 2009). BED: binge-eating disorder; 1 = strong quality; 2 = moderate quality; $3=$ weak quality 